\title{
Nonlinear optical mechanism of forming periodical nanostructures in large bandgap dielectrics
}

\author{
R. Grunwald ${ }^{1}$, S. K. Das ${ }^{1}$, A. Debroy ${ }^{1}$, E. McGlynn ${ }^{2}$, and H. Messaoudi ${ }^{1}$ \\ ${ }^{1}$ Max Born Institute Nonlinear Optics and Short-Pulse Spectroscopy, Max-Born-Strasse 2a, D-12489 Berlin, Germany \\ ${ }^{2}$ School of Physical Sciences, National Centre for Plasma Science and Technology, Dublin City University, Glasnevin, Dublin 9, Ireland \\ grunwald@mbi-berlin.de
}

\begin{abstract}
Nonlinear excitation mechanisms of plasmons and their influence on femtosecond-laser induced sub-wavelength ripple generation on dielectric and semiconducting transparent materials are discussed. The agreement of theoretical and experimental data indicates the relevance of the model.
\end{abstract}

\section{Introduction}

As is widely accepted, the formation of laser-induced periodic surface structures (LIPSS) with periods close to the wavelength can successfully be described by the Drude-Sipe theory including index changes, polarization dependent feedback and interference [1-3]. The physical mechanisms which are responsible for inducing much smaller periods on the deep-sub-wavelength scale, however, are still under discussion. Recently we also brought nonlinear-optical effects into play [4,5]. Whereas a number of experimental findings indicate a significant contribution of collective free carrier oscillations, surface plasmon polaritons (SPPs) [6-9], other observations suggest that there might be more than a single mechanism involved in the structuring processes. There are good reasons to enquire about the role of self-organized structure formation on surfaces far from thermal equilibrium, including long-range material transport which dominates on a time scale beyond the excitation time scale [10-11], or accumulation of nonthermal bonding-structure change. For these reasons, it is of importance to develop reasonable strategies for the separation of channels and the experimental verification of the specific theoretical approaches.

The studies presented here are focused on the intensity dependent creation of charge density waves in the first stage of ripple formation. Practical implications are discussed.

\section{Quantitative estimations with the extended Drude-Sipe model}

The basic assumption of our model is a multiphoton excitation of a non-metallic (dielectric or semiconducting) material followed by an ultrafast energy transfer to SPPs. SPPs start from sufficiently dense scattering features at the surface that match the dispersion relations for a given $k$-vector (spatial frequency and orientation). The interference of SPPs with each other and/or with the light wave leads to the creation of periodic intensity gratings which are inversely transferred into surface profiles by ablation and evolve with the number of pulses. In our analytical approach, however, we neglect all spectrally selective feedback processes following the appearance of the first ripples such as scattering, waveguiding, field enhancement, plasmon recombination or grating-induced redshift. Using a custom MatLab program, numerical estimations of intensity-dependent parameters were performed for a central wavelength of $800 \mathrm{~nm}$. After determining the carrier density from the peak fluence, the corresponding variation of the real part of the dielectric function and the wavelengths of the SPPs were estimated for different materials.

Figure 1 shows the result of numerical simulations for $\mathrm{ZnO}$ (black solid line) in comparison to selected experimental data (red squares). The point indicated by a black arrow corresponds to the ripples in the central region of the structured area in Figure 2. From the results it becomes obvious that the transition between high and low spatial frequency regions is not abrupt but strongly nonlinear; the fluence-dependent change of the SPP period is much more moderate towards larger periods. The comparison of the central and outer parts in Figure 2 does not support the hypothesis of bifurcations being responsible for the higher spatial frequencies under the chosen conditions. The period of high spatial frequency LIPSS, on the other hand, depends more sensitively on changes in the fluence. This and the fact that the smallest ripples appear at the low-intensity wings of the laser beam profile leads to the assumption that both the influence of intensity noise and surface roughness should be much higher in this case and the demands on the uniformity of laser and substrate are much more stringent. 


\section{Conclusions}

To conclude, the investigations give evidence for a significant contribution of nonlinear channels to the excitation of SPPs, for transparent large-bandgap materials, in particular for the initial phase of laser-induced surface structuring. Under the experimental conditions studied here ( $800 \mathrm{~nm}$ excitation of $\mathrm{ZnO})$, a continuous change of the SPP period with the fluence is found. The assumption of bifurcations is not supported, however the fast variation of the period at smaller periods could be responsible for the impression of bifurcations in some cases. Furthermore, the theory reveals that it should be possible to generate extremely small periods. However the limits of the validity of the applied models at a time scale far beyond the initial phase, at large pulse numbers and at much shorter pulse duration, have to be carefully determined in future studies. It is expected that a better control of the elementary processes of the self-organization investigated here will lead to practical implications for new nanomachining technologies.

The authors thank T. Elsaesser for stimulating discussions and M. Tischer for technical assistance. The work was financially supported by the DFG (project GR 1782/13-1) and by DAAD (2-month student's internship).

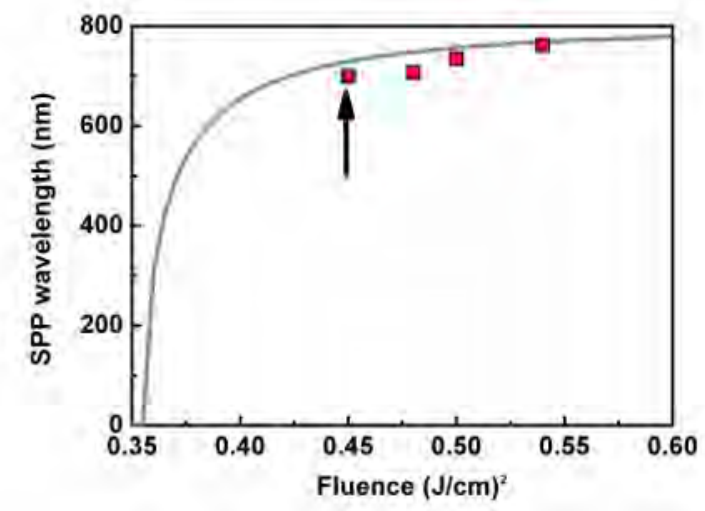

Fig 1. Theoretically estimated spatial period of surface plasmon polaritons in $\mathrm{ZnO}$; gray solid line: simulations performed for excitation@800 nm); red squares: experimental results; the point marked by the black arrow corresponds to the data derived from ripple pattern shown in Fig. 2. Obviously, a continuous change of the spatial frequency happens rather than an abrupt transition.

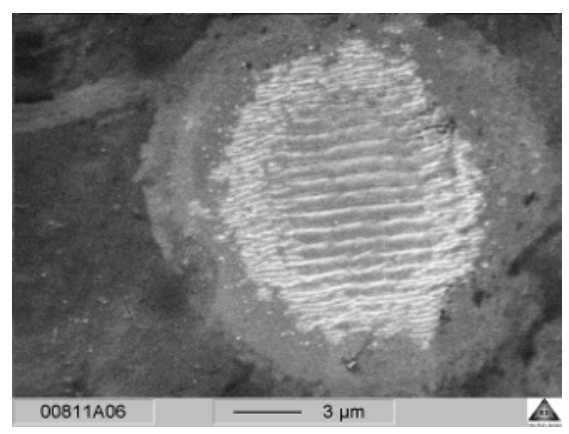

Fig 2. LIPSS obtained at the central wavelength of a Ti:sapphire laser $(800 \mathrm{~nm})$ and a peak fluence of $0.45 \mathrm{~J} / \mathrm{cm}^{2}$. The low spatial frequency part in the central region has an average period of $799 \mathrm{~nm}$. The high spatial frequency LIPSS in the outer part can not simply be explained by bifurcations (because the periods are too small, nd there is no frequency doubling).

\section{References}

[1] J. E. Sipe, J. F. Young, J. S. Preston, and H. M. van Driel, "Laser Induced periodic structures, I. Theory," Phys. Rev. B 27, $1141-1154$ (1983). [2] T. J.-Y. Derrien, R. Torres, T. Sarnet, M. Sentis, and T. E. Itina, "Formation of femtosecond laser induced surface structures on silicon: Insights from numerical modeling and single pulse experiments," Appl. Surf. Sci. 258, 9487-9490 (2012).

[3] J. Z. P. Skolski, G. R. B. E. Römer, J. V. Obona, V. Ocelik, A. J. Huis in `t Veld, and J. Th. M. De Hosson, "Laser-induced periodic surface structures: Fingerprints of light localization," Phys. Rev. B 85, 075320-1 (2912).

[4] R. Grunwald, U. Neumann, W. Seeber, H. Lange, J.-P. Mosnier, R. O'Haire, and E. McGlynn, Growth-controlled and laser-induced nanostructures in thin nonlinear-optical ZnO layers, Europ. J. Glass Sci. Technol. Pt. B: Physics and Chemistry of Glasses 48, 134-137 (2007).

[5] D. Dufft, A. Rosenfeld, S. K. Das, R. Grunwald, and J. Bonse, "Femtosecond laser-induced periodic surface structures revisited: a comparative study on ZnO," J. Appl. Phys. 105, 034908 - 034908-9 (2009).

[6] Y. Yuan, L. Jiang, X. Li, C. Wang, H. Xiao, Y. Lu, and H. Tsai, "Formation mechanisms of sub-wavelength ripples during femtosecond laser pulse train processing of dielectrics," J. Phys. D: Appl. Phys. 45, 175301 (2012).

[7] M. Huang, F. Zhao, Y. Cheng, N. Xu, and Z. Xu, "Origin of laser-induced near-subwavelength ripples; interference between surface plasmons and incident laser," ACS Nano 3, 4062-4070 (2009)

[8] S. Bashir, M. S. Rafique, and W. Husinsky, "Femtosecond laser-induced subwavelength ripples on Al, Si, CaF2 and CR-39," Nucl. Instrum. Methods in Phys. Res. B 275, 1-6 (2012).

[9] F. Garrelie, J. P. Colombier, F. Pigeon, S. Tonchev, N. Faure, M. Bounhalli, S. Reynaud, and O. Parriaux, "Evidence of surface plasmon resonance in ultrafast laser-induced ripples," Opt. Express 19, 9043 (2011).

[10] J. Reif, F. Costache, O. Varlamova, G. Jia, and M. Ratzke, "Self-organized regular surface patterning by pulsed laser ablation," Phys. Status Solidi C 6, 681-686 (2009).

[11] J. Reif, F. Costache, M. Henyk, and S. Pandelov, "Ripples revisited: non-classical morphology at the bottom of femtosecond laser ablation craters in transparent dielectrics," Appl. Surf. Sci. 197-198, 891-895 (2002). 